\title{
RELATIONS AMONGST TODA BRACKETS AND THE KERVAIRE INVARIANT IN DIMENSION 62
}

\author{
M. G. BARRATT, J. D. S. JONES AND M. E. MAHOWALD
}

\section{Introduction}

In this paper we use relations amongst Toda brackets and a lot of detailed information about the homotopy groups of spheres to show that there exists a 62-dimensional framed manifold with Kervaire invariant one. This paper, together with $[4,5]$, represents an effort to supply full details for a number of the results announced in [11], and to explore further some of the ideas behind that paper.

The general problem of whether or not there are elements $\theta_{n} \in \pi_{2^{n+1}-2}^{s}$ with nonzero Kervaire invariant will not be solved by the methods of this paper. The first three cases of this problem are trivial: $\theta_{1}=\eta^{2}, \theta_{2}=v^{2}, \theta_{3}=\sigma^{2}$, where $\eta, v$ and $\sigma$ are the three Hopf maps. It is known that $\theta_{4}$ exists and indeed $\theta_{4}$ is reasonably well understood $[13,16,8]$. The point of this paper is to construct another non-obvious example: $\theta_{5} \in \pi_{62}^{s}$. However not even the most optimistic of us would claim that $\theta_{5}$ is well understood.

We begin this paper with some preliminaries on cell diagrams and Toda brackets. In $\S 2$ we outline the proof of the existence of $\theta_{5}$, and in $\S \S 3$ and 4 we give the detailed calculations needed to complete this proof. These calculations require constant references to $[3,6,13,14,20,21]$ for information about $\pi_{*}^{s}$ and the $E_{2}$ and $E_{\infty}$ terms of the mod 2 Adams spectral sequence. Some of this information is also contained in $[10,16]$ and the tables at the end of [22]. There is an appendix with a proof of a general result on the vanishing of certain Toda brackets; a special case of this result is used in the main body of this paper.

\section{Preliminaries}

We begin with a brief discussion of cell diagrams and give two lemmas on Toda brackets.

Let $X$ be a CW complex, then a cell diagram for $X$ consists of nodes and edges. The nodes are in one-one correspondence with the cells of $X$ and may be labelled with symbols to indicate the dimensions of the cells. When two nodes are joined by an edge as in Figure 1, then it is possible to form a subquotient

$$
X^{\prime} / X^{\prime \prime}=S^{n} \cup_{f} e^{m}
$$

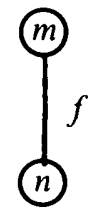

FIG. 1

Received 19 November, 1982; revised 8 December, 1983 and 27 February, 1984.

1980 Mathematics Subject Classification 55Q45. 
such that the cells which are in the subcomplex $X^{\prime}$ but not in $X^{\prime \prime}$ are just those corresponding to the two nodes, and such that the attaching map is the map $f$ shown. Suppose we have two nodes labelled $n$ and $m$ with $n<m$ and there is no edge joining them. Then there are two cases.

(i) There is an integer $k \geqslant 2$ and a sequence of nodes labelled $n_{i}, 0 \leqslant i \leqslant k$, with $n=n_{0}<n_{1}<\ldots<n_{k}=m$ and edges joining the node $n_{i}$ to the node $n_{i+1}$. In this case we do not assert that there is a subquotient of the form (1.1); this does not imply that there is no such subquotient.

(ii) There is no sequence of nodes as in (i). Then we assert that there exists a subquotient of the form (1.1) with

$$
X^{\prime} / X^{\prime \prime}=S^{n} \vee S^{m}
$$

For example consider the complex $X=S^{n} \cup e^{m} \cup e^{l}$. Then a cell diagram for $X$ is given in Figure 2, and this simply tells us that $X=\left(S^{n} \cup{ }_{f} e^{m}\right) \cup{ }_{g^{\prime}} e^{l}$ where, if

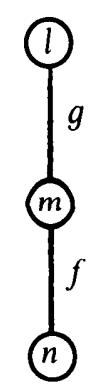

FIG. 2

$p: S^{n} \cup{ }_{f} e^{m} \rightarrow S^{m}$ is the collapsing map, then $g^{\prime} p=g$. This is the best we can do without further information; however, if it happens that $f$ is trivial or $g$ is trivial then, respectively, $X$ admits the cell diagrams of Figure 3.

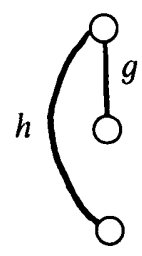

FIG. 3

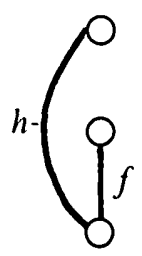

We are usually only interested in complexes up to homotopy type so from now on we use the phrase 'admits a cell diagram' to mean that there is a complex homotopy equivalent to $X$ with the given cell diagram, and we label the edges by the homotopy classes of the appropriate maps. We can also form stable cell diagrams for stable complexes and, in an analogous manner, cell diagrams for maps.

We always regard relations amongst Toda brackets as conditions for the existence of complexes with specified cell diagrams. For example, Toda's relation 


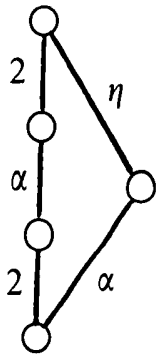

FIG. 4

that if $\alpha \in \pi_{n}^{s}$ and $2 \alpha=0$ then $\eta \alpha \in\langle 2, \alpha, 2\rangle$ shows that there is a stable complex with the cell diagram of Figure 4.

We come now to our lemmas on Toda brackets.

LeMmA 1.2. Suppose that $\alpha \in \pi_{a}^{s}, \beta \in \pi_{b}^{s}, \gamma \in \pi_{c}^{s}$, then necessary and sufficient conditions for forming the Toda bracket $\langle\alpha, \beta, \alpha, \gamma\rangle$ are that

(i) $\alpha \beta=\alpha \gamma=0$,

(ii) $0 \in\langle\alpha, \beta, \alpha\rangle, 0 \in\langle\beta, \alpha, \gamma\rangle$.

Proof. Suppose we are given maps

$$
S^{0} \stackrel{f}{\longleftarrow} S^{a} \stackrel{g}{\longleftarrow} S^{a+b} \stackrel{h}{\longleftarrow} S^{2 a+b} \stackrel{k}{\longleftarrow} 2^{2 a+b+c}
$$

representing the stable homotopy classes $\alpha, \beta, \alpha, \gamma$ respectively. We begin by choosing null homotopies $g h \simeq 0, h k \simeq 0$ such that the resulting representative for $\langle\beta, \alpha, \gamma\rangle$ is null-homotopic. The whole indeterminacy of $\langle\alpha, \beta, \alpha\rangle$ is

$$
\alpha \pi_{2 a+b+1}^{s}\left(S^{a}\right)+\pi_{a+b+1}^{s}\left(S^{0}\right) \alpha=\pi_{a+b+1}^{s}\left(S^{0}\right) \alpha,
$$

so that we may now choose a null-homotopy $f g \simeq 0$ such that the representative for the Toda bracket $\langle\alpha, \beta, \alpha\rangle$ obtained by using this null-homotopy of $f g$ and our given null-homotopy of $g h$ is null-homotopic. Therefore we may form the bracket $\langle\alpha, \beta, \alpha, \gamma\rangle$.

Lemma 1.3. Suppose that $\alpha \in \pi_{2 n}^{s}$ and $2 \alpha=0$, then $0 \in\langle\alpha, 2, \alpha\rangle$.

There are quick proofs available for this lemma; however, we choose to present it as a corollary of a more general result which we discuss in detail in an appendix.

2. The Kervaire invariant in dimension 62 - an outline of the argument

We use the usual notation, $\phi_{n, n}$, for the secondary mod 2 cohomology operation based on the Adem relation

$$
S q^{2^{n}} S q^{2^{n}}=\sum_{i=0}^{n-1} S q^{2^{n+1}-2 i} S q^{2^{i}}
$$


Our object is to construct $\theta_{5} \in \pi_{62}^{s}$ detected by $\phi_{5,5}$. From now on we work stably and at the prime 2 so we shall now write $\pi_{j}$ for the 2-primary component of $\pi_{j}^{s}$; all cohomology groups are to have mod 2 coefficients.

To construct $\theta_{n} \in \pi_{2^{n+1}-2}$ detected by $\phi_{n, n}$ we can try to construct a complex $X$ and maps

$$
f: S^{2 n+1-2} \longrightarrow X, \quad g: X \longrightarrow S^{0}
$$

satisfying the following conditions.

(i) Both $f$ and $g$ are zero in $\bmod 2$ cohomology.

(ii) For $i \leqslant n-1$ the functional cohomology operations

are all zero.

$$
S q_{g}^{2^{i}}: H^{0} S^{0} \longrightarrow H^{2^{i}-1} X
$$

(iii) $S q_{g}^{2^{n}}: H^{0} S^{0} \rightarrow H^{2^{n}-1} X$ is non-zero.

(iv) The functional cohomology operation $S q_{f}^{2^{n}}$ is defined and non-zero on $S q_{g}^{2^{n}} H^{0} S^{0} \subseteq H^{2^{n}-1} X$.

If $X, f$ and $g$ satisfy these conditions the Peterson-Stein formula $[17,19]$ shows that $g f$ is detected by $\phi_{n, n}$. Note that this is not the only way in which we can use the Peterson-Stein formula to prove that a composite $g f$ is detected by $\phi_{n, n}$-it is simply the one which occurs in the examples we discuss.

TheOREM 2.1. There are a stable complex $X$ and maps $f: S^{62} \rightarrow X \quad g: X \rightarrow S^{0}$ with the cell diagram of Figure 5 for some $\psi_{1}, \psi_{2} \in \pi_{32}, \psi_{3} \in \pi_{44}, \psi_{4} \in \pi_{46}$. The maps

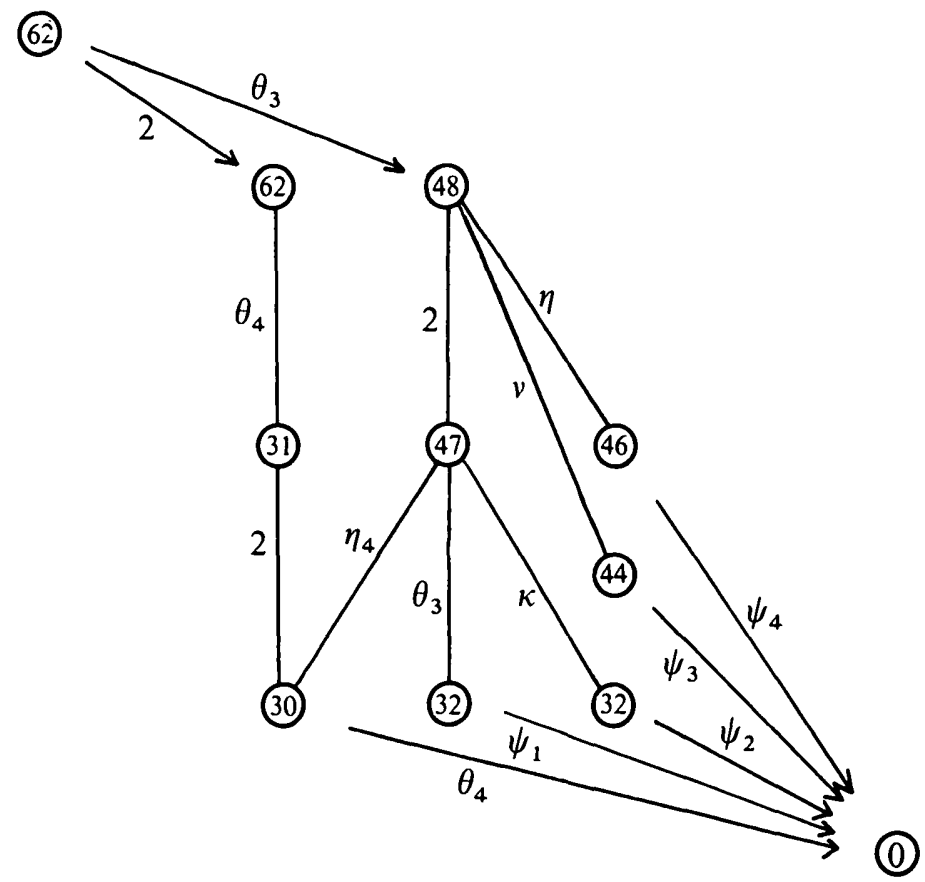

FiG. 5 
$f$ and $g$ satisfy the hypotheses (i)-(iv) above with $n=5$ so that the composite $g f$ is $\theta_{5} \in \pi_{62}$.

The notation for homotopy classes is that of $[\mathbf{2 2}, 3]$ with the minor change that here we choose to write $\eta_{j} \in \pi_{2 j}$ [12] for the element called $n_{j-1}$ in [3]. Thus $\eta, v$ and $\sigma$ are the Hopf maps in $\pi_{1}, \pi_{3}$ and $\pi_{7}, \theta_{3}=\sigma^{2}, \theta_{4}$ is the unique non-trivial element of $\pi_{30}=\mathbb{Z} / 2$ [13], and $\kappa$ is the element of $\pi_{14}$ described in Toda's book.

In order to give some kind of motivation for this construction we go back to explain a construction of $\theta_{4}$. Suppose that we can form the Toda bracket $\left\langle\theta_{3}, 2, \theta_{3}, 2\right\rangle$, then any element in this set factors as

$$
S^{30} \stackrel{f}{\longrightarrow} Y \stackrel{g}{\longrightarrow} S^{0}
$$

with the cell diagram of Figure 6.

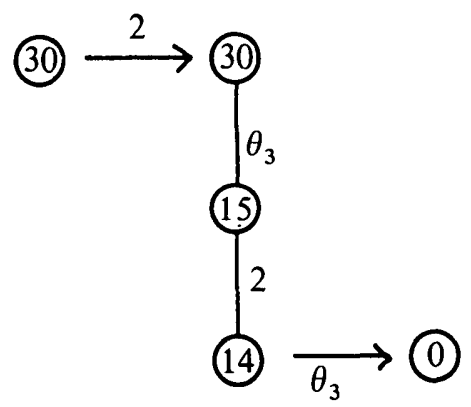

FiG. 6

Set $n=4$, then evidently $f$ and $g$ satisfy conditions (i) and (ii). Adams's decomposition of $S q^{16}$ [1], shows that $f$ and $g$ satisfy (iii) and (iv) so that $g f=\theta_{4}$. According to Lemma 1.2 the conditions for forming this bracket are

(a) $2 \theta_{3}=0$,

(b) $0 \in\left\langle 2, \theta_{3}, 2\right\rangle$,

(c) $\mid 0 \in\left\langle\theta_{3}, 2, \theta_{3}\right\rangle$.

Now (a) is trivial. By Toda's formula $\eta \theta_{3} \in\left\langle 2, \theta_{3}, 2\right\rangle$, and $\eta \theta_{3}=0$ (compare [22]) so that (b) follows, and finally (c) follows from Lemma 1.3.

We now try and repeat this argument with $\theta_{3}$ replaced by $\theta_{4}$. We have $2 \theta_{4}=0$ by [13] and so Lemmas 1.2 and 1.3 show that the only obstruction to forming $\left\langle\theta_{4}, 2, \theta_{4}, 2\right\rangle$ is the set $\eta \theta_{4}+2 \pi_{31}$. However $\eta \theta_{4}$ is a non-zero element of $\pi_{31}$ of minimal Adams filtration and so cannot be in $2 \pi_{31}$. Therefore there is no map with the cell diagram of Figure 7. 


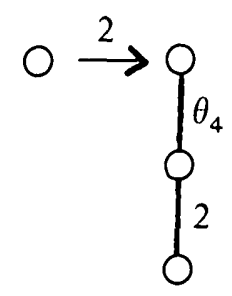

F1G. 7

However the relation $\eta \theta_{4} \in\left\langle 2, \theta_{4}, 2\right\rangle$ shows that there is a map with the cell diagram of Figure 8 , but we meet the obstruction $\theta_{4}^{2}$ to finding a map of this 4-cell

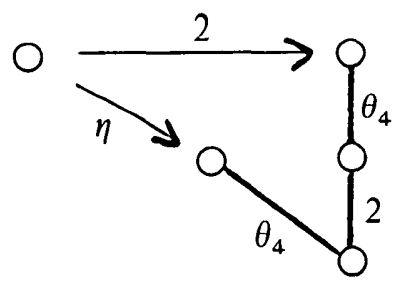

FIG. 8

complex which restricts to $\theta_{4}$ on the bottom cell. We do not prove that $\theta_{4}^{2}=0$ as this requires substantial further calculations; anyway if we did we would then use a better line of argument which shows that $\theta_{5}$ exists and $2 \theta_{5}=0[11,4]$. We avoid this problem by using a different decomposition of $\eta \theta_{4}[3,3.2 .1]$ :

$$
\eta \theta_{4} \in\left\langle\eta_{4}, 2, \theta_{3}\right\rangle \text {. }
$$

Thus we can find a map with the cell diagram of Figure 9, and this is the beginning of Figure 5. Now we deal with the obstructions to find a map of this 5-cell complex which restricts to $\theta_{4}$ on the bottom cell.

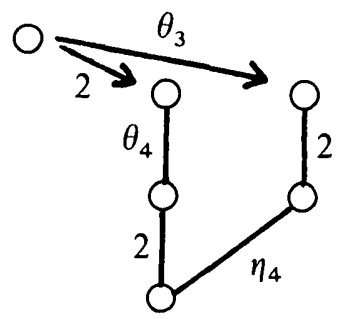

FIG. 9 
Lemma 2.3. There are elements $\psi_{1}, \psi_{2} \in \pi_{32}$ such that

(i) $\eta \psi_{1}=0$,

(ii) $\theta_{4} \eta_{4}=\psi_{1} \theta_{3}+\psi_{2} \kappa$.

LeMMA 2.4. (i) $\theta_{4}\left\langle\eta, 2, \eta_{4}\right\rangle=0$,

(ii) Suppose that $\psi_{1} \in \pi_{32}$ is such that $\eta \psi_{1}=0$, then $\psi_{1}\left\langle\eta, 2, \theta_{3}\right\rangle=0$,

(iii) $0 \in\langle\eta, 2, \kappa\rangle$.

LEMMA 2.5. Let $a$ be any element of $\pi_{47}$ such that $\eta a=0$. Then there exist $b \in \pi_{47}, c \in \pi_{46}, d \in \pi_{44}$ such that $a=2 b+\eta c+v d$.

Assuming these three lemmas, we complete the proof of Theorem 2.1. From Lemmas 1.3 and 2.3 there is a complex $X_{0}$ and a map $g_{0}: X_{0} \rightarrow S^{0}$ with the cell diagram of Figure 10, where $\psi_{1}$ and $\psi_{2}$ are chosen to satisfy the conclusions of

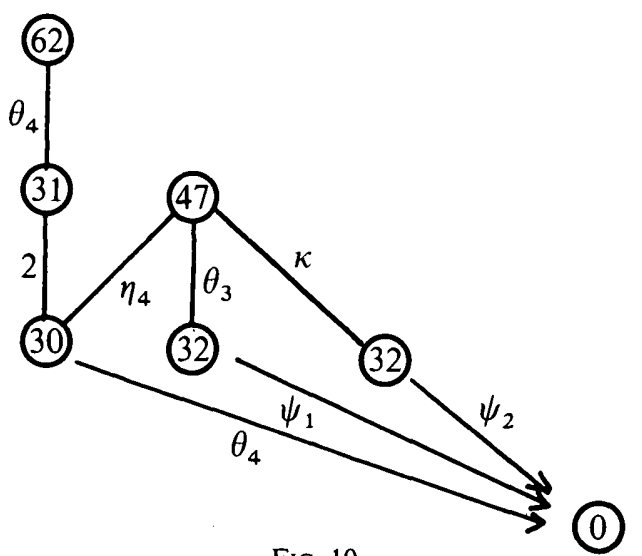

FIG. 10

Lemma 2.3. There is a map $h: S^{47} \rightarrow X_{0}$ of degree 2 in integral homology, and we now deal with the obstruction to extending $g_{0}$ over the cofibre of $h$. Write

$$
\begin{aligned}
& \delta_{1}=\left(\theta_{4}, \psi_{1}, \psi_{2}\right): S^{30} \vee S^{32} \vee S^{32} \longrightarrow S^{0}, \\
& \delta_{2}=\left(\begin{array}{c}
\eta_{4} \\
\theta_{3} \\
\kappa
\end{array}\right): S^{46} \longrightarrow S^{30} \vee S^{32} \vee S^{32},
\end{aligned}
$$

then this obstruction is the set

$$
\left\langle\delta_{1}, \delta_{2}, 2\right\rangle \subseteq \pi_{47}
$$

We aim to use Lemma 2.5 , so multiply by $\eta$ :

$$
\left\langle\delta_{1}, \delta_{2}, 2\right\rangle \eta=\delta_{1}\left\langle\delta_{2}, 2, \eta\right\rangle \subseteq \theta_{4}\left\langle\eta_{4}, 2, \eta\right\rangle+\psi_{1}\left\langle\theta_{3}, 2, \eta\right\rangle+\psi_{2}\langle\kappa, 2, \eta\rangle .
$$


We check indeterminacy to see that (2.6) is actually an equality. The indeterminacy of the right-hand side is

$$
\theta_{4} \eta_{4} \pi_{2}+\theta_{4} \eta \pi_{17}+\psi_{1} \theta_{3} \pi_{2}+\psi_{1} \eta \pi_{15}+\psi_{2} \kappa \pi_{2}+\psi_{2} \eta \pi_{15}
$$

Now since $\pi_{2}$ is generated by $\eta^{2}$ this reduces to

$$
\theta_{4} \eta \pi_{17}+\psi_{1} \eta \pi_{15}+\psi_{2} \eta \pi_{15}
$$

and this is equal to the indeterminacy of the left hand side.

From Lemma 2.4 the right-hand side of (2.6) contains zero. Therefore $\left\langle\delta_{1}, \delta_{2}, 2\right\rangle$ contains an element $a$ such that $\eta a=0$; its indeterminacy contains $2 \pi_{47}$ and so by Lemma 2.5 it contains an element of $v \pi_{44}+\eta \pi_{46}$. We have now proved that there exists a complex $X$ and map $g: X \rightarrow S^{0}$ with the cell diagram of Figure 5 in Theorem 2.1; the primary obstructions $2 \theta_{4}, 2 \eta_{4}, 2 \theta_{3}, 2 \kappa, \theta_{4} \eta_{4}-\psi_{1} \theta_{3}-\psi_{2} \kappa$ all vanish, and the secondary obstructions $\left\langle\theta_{4}, 2, \theta_{4}\right\rangle$ and $\left\langle\delta_{1}, \delta_{2}, 2\right\rangle+v \pi_{44}+\eta \pi_{46}$ both contain zero.

Next we consider the obstructions to the existence of a map $f: S^{62} \rightarrow X$ with cell diagram given in Figure 5. The primary obstructions $2 \theta_{4}, 2 \theta_{3}, \eta \theta_{3}, v \theta_{3}$ all vanish. The secondary obstructions are the sets

$$
\left\langle 2, \theta_{4}, 2\right\rangle-\left\langle\eta_{4}, 2, \theta_{3}\right\rangle \quad\left\langle\theta_{3}, 2, \theta_{3}\right\rangle \quad\left\langle\kappa, 2, \theta_{3}\right\rangle .
$$

The first contains zero by (2.2), and the other two contain zero since $\pi_{29}=0$. Indeed the fact that $\pi_{29}=0$ shows that there is no difficulty over choices of nullhomotopies, or co-extensions, needed to ensure more than one outcome.

It is straightforward, using Adams's decomposition of $S q^{32}$, to check that $f$ and $g$ satisfy conditions (i)-(iv) with $n=5$, so that $g f=\theta_{5} \in \pi_{62}$.

\section{Some homotopy groups of spheres}

In this section we discuss the 45,46 and 47 stems and prove Lemma 2.5. We use the Adams spectral sequence with $E_{2}$ term

$$
E_{2}^{s, t}=\operatorname{Ext}_{A}^{s, t}(\mathbb{Z} / 2, \mathbb{Z} / 2)
$$

where $A$ is the mod 2 Steenrod algebra. Diagram A is the usual kind of diagram of this spectral sequence; vertical lines represent multiplication by $h_{0}$, lines sloping to the right represent multiplication by $h_{1}$, and lines sloping to the left are differentials $\partial_{r}$. The chart is taken from Tangora's table [20].

The differentials defined on the 45 and 46 stems are obvious deductions from 1.1.4, 1.1.5 and 8.9 of [13]. The differentials on the elements $h_{0}^{i} Q^{\prime}$ are determined by consideration of the image of $J[2,9]$. They are given by

$$
\partial_{2} Q^{\prime}=h_{0} P^{2} r=P^{2} s, \quad \partial_{3} h_{0}^{5} Q^{\prime}=h_{0} P^{4} d_{0}
$$

(recall that $h_{0} r=s$ ). Next note that $h_{1} P^{1} u$ is a product of infinite cycles, and so is an infinite cycle; therefore $P^{2} d_{0} e_{0}$ is non-zero in $E_{4}$ and $[13,1.1 .4]$ shows that $\partial_{4} P^{2} d_{0} e_{0}=P^{4} d_{0}$. 


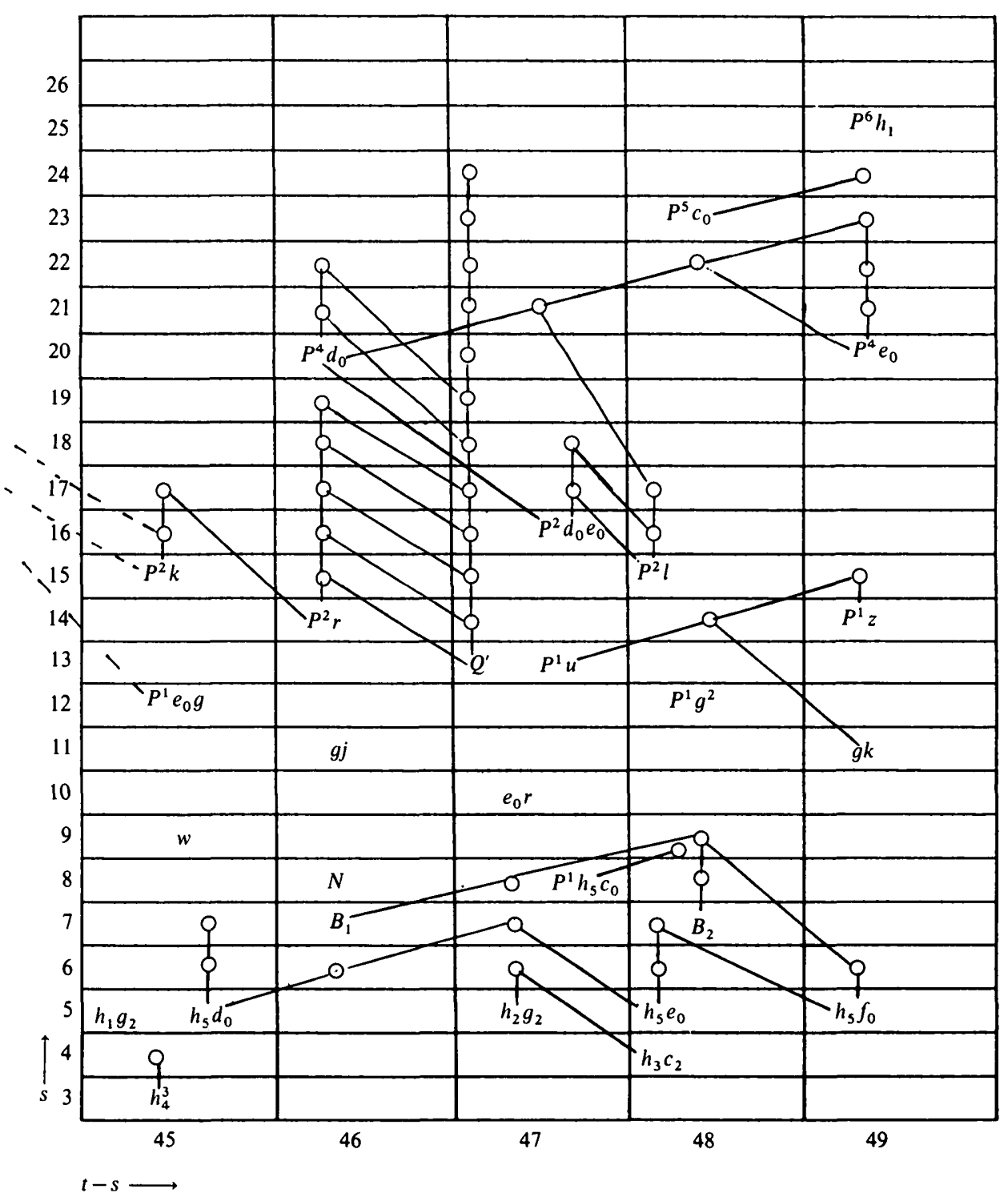

Diagram A

If $a \in E_{2}$ is an infinite cycle then we use the usual notation that $\{a\} \subseteq \pi_{*}$ denotes the set of homotopy classes detected by $a$. It can happen that $0 \in\{a\}$; this is the case if and only if $a$ is a boundary. We usually write $\{a\}=\alpha$ when there is a unique element $\alpha$ in the set $\{a\}$; a similar convention applies to a Toda bracket with a unique element.

We now prove that $h_{5} P^{1} c_{0}$ is an infinite cycle. Let $\rho_{15} \in \pi_{15}$ be a generator of the image of $J$, so that $\eta \rho_{15} \in \pi_{16}$ generates the image of $J$ and $\eta \rho_{15} \in\left\{P^{1} c_{0}\right\}$, compare [3]. Form the Toda bracket $\left\langle\theta_{4}, 2, \eta \rho_{15}\right\rangle$. Now $\theta_{4} \in\left\{h_{4}^{2}\right\}, \partial_{2} h_{5}=h_{0} h_{4}^{2}$ and so the theorem of Moss [18] relating Toda brackets in $\pi_{*}$ and Massey products in the Adams spectral sequence shows that there is an element of $\left\langle\theta_{4}, 2, \eta \rho_{15}\right\rangle$ detected by $h_{5} P^{1} c_{0}$, and so $h_{5} P^{1} c_{0}$ must be an infinite cycle. 
The differentials defined on the 47 stem now follow in a straightforward manner. For the differentials on the 48 and 49 stems we need some preliminary results, most of which are in Tangora's paper [21].

THEOREM 3.1. (i) $\eta\left\{h_{4}^{3}\right\} \cap\left\{B_{1}\right\} \neq \varnothing$,

(ii) $0 \notin \eta\{g j\}$,

(iii) $\eta\{g j\} \subseteq\left\{P^{1} u\right\}$,

(iv) $\eta\{w\} \subseteq\{g j\}$,

(v) $0 \notin \eta\left\{e_{0} r\right\}$,

(vi) $\eta\left\{e_{0} r\right\} \subseteq\left\{P^{1} g^{2}\right\}$.

The proofs of (i)-(iv) are in part 2 of [21]; (v) is proved in [7, Proposition 4.2] using the relation $g k=e_{0} l[\mathbf{2 0}]$ and the differential $\partial_{3} g k=h_{1} P^{1} u[13,1.1 .4]$. Finally (vi) is the only possibility, given (v).

Lemma 3.2. (i) $\theta_{4} \kappa=0$,

(ii) $\left\langle 2, \theta_{4}, \kappa\right\rangle \cap\left\{h_{5} d_{0}\right\} \neq \varnothing$.

Proof. (i) We know that $\theta_{4} \in\left\{h_{4}^{2}\right\}$ and $\kappa \in\left\{d_{0}\right\}$ and so we check the product $h_{4}^{2} d_{0} \in \mathrm{Ext}_{A}(\mathbb{Z} / 2, \mathbb{Z} / 2)$; from $[20], h_{4} d_{0}=0$ since it lies in a zero group. Therefore $\kappa \theta_{4}$ has filtration $\geqslant 7$ in $\pi_{44}$ and from $E_{\infty}$ of the spectral sequence [13] it follows that $\kappa \theta_{4}=0$.

(ii) This follows from Moss's theorem and the differential $\partial_{2} h_{5}=h_{0} h_{4}^{2}$.

THEOREM 3.3. (i) $4\left\{h_{4}^{3}\right\} \subseteq\left\{h_{0} h_{5} d_{0}\right\}$,

(ii) $\pi_{45}=\mathbb{Z} / 16 \oplus \mathbb{Z} / 2 \oplus \mathbb{Z} / 2 \oplus \mathbb{Z} / 2$.

(iii) It is possible to pick $\alpha \in\left\{h_{4}^{3}\right\}, \beta \in\left\langle 2, \theta_{4}, \kappa\right\rangle \cap\left\{h_{5} d_{0}\right\}, \gamma \in\left\{g_{2}\right\} \subseteq \pi_{44}$, $\delta \in\{w\}$ such that $4 \alpha=2 \beta$ and $\pi_{45}$ is generated by

$$
\begin{array}{cccc}
\alpha & \text { order } 16, & 2 \alpha-\beta & \text { order } 2, \\
\eta \gamma \quad & \text { order } 2, & \delta & \text { order } 2 .
\end{array}
$$

Proof. (i) and (ii) are proved in part 2 of [21]. To prove (iii) pick $\alpha \in\left\{h_{4}^{3}\right\}$ and $\beta \in\left\langle 2, \theta_{4}, \kappa\right\rangle \cap\left\{h_{5} d_{0}\right\}$. Then from (i) $4 \alpha-2 \beta$ has filtration $\geqslant 7$ and so there are integers $x, y$ such that $4 \alpha-2 \beta=x \delta+4 y \beta$. Multiply by $\eta$ and use Theorem 3.1(iv) to conclude that $x \delta=0$, and now replace $\alpha$ by $\alpha-y \beta$. We now get the relation $4 \alpha=2 \beta$. The rest of (iii) is trivial.

From now on we always use the notation of Theorem 3.3 for $\pi_{45}$.

Lemma 3.4.

$$
\eta^{3} \beta=0
$$


Proof. Since $\eta^{3}=4 v$ and $8 v=0$ we get $\eta^{3} \beta=4 v \beta=8 v \alpha=0$.

Next we determine the only non-obvious differential we need; this was first found by G. W. Whitehead.

LemMa 3.5 .

$$
\eta^{3}\left\{h_{4}^{3}\right\}=0 .
$$

Proof. From Theorem 3.3, $4\left\{h_{4}^{3}\right\}=4 \alpha=2 \beta$, and so the relation $\eta^{3}=4 v$ shows that $\eta^{3}\left\{h_{4}^{3}\right\}=4 v \alpha=2 v \beta$. We now show that $4 \alpha+8 \pi_{45}$ contains a multiple of $\sigma$. By Moss's theorem $\left\langle 8 \sigma, 2, \theta_{4}\right\rangle \cap\left\{h_{0}^{3} h_{3} h_{5}\right\} \neq \varnothing$. However

$$
\sigma\left\langle 8 \sigma, 2, \theta_{4}\right\rangle=\langle\sigma, 8 \sigma, 2\rangle \theta_{4}=\rho_{15} \theta_{4},
$$

where $\rho_{15} \in \pi_{15}$ generates the image of $J$. By [21, Corollary 2.8] $\rho_{15} \theta_{4} \in\left\{h_{0}^{2} h_{5} d_{0}\right\}$ and so

$$
\sigma\left\langle 8 \sigma, 2, \theta_{4}\right\rangle \subseteq\left\{h_{0}^{2} h_{5} d_{0}\right\} .
$$

From [3] two elements of $\left\{h_{0}^{3} h_{3} h_{5}\right\}$ differ by a multiple of $v^{2}$, so since $\sigma v=0$,

$$
\sigma\left\{h_{0}^{3} h_{3} h_{5}\right\}=\sigma\left\langle 8 \sigma, 2, \theta_{4}\right\rangle \subseteq\left\{h_{0}^{2} h_{5} d_{0}\right\} .
$$

One easily deduces that $\sigma\left\{h_{0}^{2} h_{3} h_{5}\right\} \subseteq\left\{h_{0} h_{5} d_{0}\right\}$, and so from Theorem 3.3, if $\xi \in\left\{h_{0}^{2} h_{3} h_{5}\right\}$, then

$$
\sigma \xi=4 \alpha+8 x \alpha+y \delta, \quad x, y \in \mathbb{Z} .
$$

But we now check that $\eta^{2} \sigma \pi_{38}=0$; from the structure of $E_{\infty}$ of the mod 2 Adams spectral sequence given in [13], taking account of the corrections in $[3,6]$, we see that $\eta^{2} \pi_{38}$ must be contained in the subgroup of $\pi_{40}$ generated by $\bar{\kappa}^{2}$ and since $\sigma \bar{\kappa}=0$ we see that $\eta^{2} \sigma \pi_{38}=0$. Therefore

$$
0=\eta^{2} \sigma \xi=y \eta^{2} \delta
$$

but since $\eta^{2} \delta \neq 0$ (Theorem 3.1), we see that $y$ is even so that $y \delta=0$. Therefore $\sigma \xi=4 \alpha+8 x \alpha$, and

$$
0=v \sigma \xi=4 v \alpha=\eta^{3}\left\{h_{4}^{3}\right\} .
$$

COROLlary 3.6.

$$
\partial_{3} h_{0} h_{5} f_{0}=h_{1}^{2} B_{1}=h_{0}^{2} B_{2} .
$$

Proof. By Theorem 3.1 there exists an element $\xi \in\left\{h_{4}^{3}\right\}$ such that $\eta \xi \in\left\{B_{1}\right\}$ and so by Lemma 3.5 ,

$$
0=\eta^{3} \xi \in\left\{h_{1}^{2} B_{1}\right\} .
$$

Therefore $h_{1}^{2} B_{1}$ is a boundary and the only possibility is that

$$
\partial_{3} h_{0} h_{5} f_{0}=h_{1}^{2} B_{1} .
$$

This proves the corollary. The differentials on the 48 and 49 stems follow. 
We now establish Lemma 2.5. The 47 stem is generated by the elements

$$
\begin{aligned}
& \nu \gamma \in\left\{h_{2} g_{2}\right\}, \quad \eta^{2} \alpha \in\left\{h_{1} B_{1}\right\}, \quad x \in\left\{P^{1} h_{5} c_{0}\right\}, \\
& y \in\left\{e_{0} r\right\}, \quad \eta^{2} \delta \in\left\{P^{1} u\right\}, \quad \rho_{47} \in\left\{h_{0}^{7} Q^{\prime}\right\},
\end{aligned}
$$

where $\rho_{47}$ is a generator of the image of $J$.

Now $\eta x \in\left\{h_{1} P^{1} h_{5} c_{0}\right\}$ and, in view of Corollary 3.6, $h_{1} P^{1} h_{5} c_{0}$ is not a boundary. By Theorem 3.1 $\eta y \in\left\{P^{1} g^{2}\right\}$; from the properties of the image of $J$ [2] we know that $\eta \rho_{47}$ is non-zero, and therefore $\eta \rho_{47} \in\left\{P^{5} c_{0}\right\}$. It now follows that $\eta x, \eta y$ and $\eta \rho_{47}$ are independent over $\mathbb{Z} / 2$. However $\eta v \gamma=0$ (since $\eta v=0$ ), $\eta^{3} \alpha=0$ (see Lemma 3.5) and $\eta^{3} \delta=0$ (since $\eta^{3}=4 v$ and $2 \delta=0$ ) and therefore the kernel of $\eta: \pi_{47} \rightarrow \pi_{48}$ is generated by $v \gamma, \eta^{2} \alpha, \eta^{2} \delta$ and $2 \rho_{47}$. This completes the proof.

\section{Final proofs}

We finish by proving Lemmas 2.3 and 2.4; we begin with Lemma 2.4.

Proof of Lemma 2.4. (i) Pick $v^{*} \in\left\{h_{2} h_{4}\right\} \subseteq \pi_{18}$; let $\mu_{9} \in \pi_{9}, \mu_{17} \in \pi_{17}$ be the unique elements of the Toda brackets $\langle\eta, 2,8 \sigma\rangle,\left\langle\mu_{9}, 2,8 \sigma\right\rangle$. Then $\pi_{18}=\mathbb{Z} / 8 \oplus \mathbb{Z} / 2$ is generated by $v^{*}$, of order 8 , and $\eta \mu_{17}$, of order 2 . Since $\eta_{4} \in\left\{h_{1} h_{4}\right\}$ Moss's theorem shows that the sets $\left\langle\eta, 2, \eta_{4}\right\rangle$ and $\left\{\left\langle h_{1}, h_{0}, h_{1} h_{4}\right\rangle\right\}$ have a common element, however

$$
\left\langle h_{1}, h_{0}, h_{1} h_{4}\right\rangle=\left\langle h_{1}, h_{0}, h_{1}\right\rangle h_{4}=h_{0} h_{2} h_{4},
$$

and a check on indeterminacy shows that

$$
\left\langle\eta, 2, \eta_{4}\right\rangle \subseteq\left\{2 v^{*}, 2 v^{*}+\eta \mu_{17}\right\}+4 \pi_{18}
$$

Therefore $\theta_{4}\left\langle\eta, 2, \eta_{4}\right\rangle \subseteq\left\{0, \eta \theta_{4} \mu_{17}\right\}$ and we now manipulate Toda brackets to show that $\eta \theta_{4} \mu_{17}=0$ :

$$
\left.\begin{array}{rlrl}
\eta \theta_{4} \mu_{9} & \in \eta \theta_{4}\langle\eta, 2,8 \sigma\rangle & & \\
& =\left\langle\eta \theta_{4}, \eta, 2\right\rangle 8 \sigma & & \text { since } \eta^{2} \theta_{4}=0 \\
& =0 & & \text { since } 8 \pi_{33}=0
\end{array}\right]
$$

(N.B. The corrections to [13] given in [3] show that $\pi_{41}=(\mathbb{Z} / 2)^{5}$.) Therefore $\theta_{4}\left\langle\eta, 2, \eta_{4}\right\rangle=0$ and this proves Lemma $2.4(\mathrm{i})$. 
(ii) We have $\psi_{1}\left\langle\eta, 2, \theta_{3}\right\rangle=\left\langle\psi_{1}, \eta, 2\right\rangle \theta_{3} \subseteq \theta_{3} \pi_{34}$ and using the table of generators of $\pi_{34}$ given in [3] we see the only product of $\theta_{3}$ with $\pi_{34}$ which is not obviously zero is

$$
\theta_{3}\left\langle\eta, 2, \eta_{5}\right\rangle=\left\langle\theta_{3}, \eta, 2\right\rangle \eta_{5} .
$$

From Toda's tables we see that composition with $\eta$ defines an injective homomorphism $\pi_{16} \rightarrow \pi_{17}$. However

$$
\left\langle\theta_{3}, \eta, 2\right\rangle \eta=\theta_{3}\langle\eta, 2, \eta\rangle=\theta_{3}\left(2 v+\eta \pi_{2}\right)=0,
$$

and therefore $\left\langle\theta_{3}, \eta, 2\right\rangle=0$. Thus $\theta_{3} \pi_{34}=0$ and so Lemma 2.4(ii) is proved.

(iii) The group $\pi_{16}=\mathbb{Z} / 2 \oplus \mathbb{Z} / 2$ is generated by $\eta_{4}$ and $\eta \rho_{15}$. The Adams filtration of any element of $\langle\eta, 2, \kappa\rangle$ is $\geqslant 3$ so $\langle\eta, 2, \kappa\rangle$ is contained in the subgroup of $\pi_{16}$ generated by $\eta \rho_{15}$; but this subgroup is the indeterminacy of $\langle\eta, 2, \kappa\rangle$ and so $\langle\eta, 2, \kappa\rangle$ contains zero.

We now start on the proof of Lemma 2.3.

Lemma 4.1. (i) $\eta_{4} \theta_{4} \in \eta\left\langle\theta_{3}, 2, \theta_{4}\right\rangle$,

(ii) $\eta \eta_{4} \theta_{4}=0$.

Proof. (i) Moss's theorem and a check on indeterminacy show that

$$
\left\langle\eta, \theta_{3}, 2\right\rangle=\left\{h_{1} h_{4}\right\} .
$$

So $\eta_{4} \in\left\langle\eta, \theta_{3}, 2\right\rangle$ and $\eta_{4} \theta_{4} \in\left\langle\eta, \theta_{3}, 2\right\rangle \theta_{4}=\eta\left\langle\theta_{3}, 2, \theta_{4}\right\rangle$. In fact one can check that there is no indeterminacy, but we do not need this.

(ii) We use 3.2.1 and 3.3.2 of [3]; $\eta \theta_{4} \in\left\langle\theta_{3}, 2, \eta_{4}\right\rangle, \eta_{4}^{2}=0$, and so

$$
\eta \eta_{4} \theta_{4} \in\left\langle\theta_{3}, 2, \eta_{4}\right\rangle \eta_{4}=\theta_{3}\left\langle 2, \eta_{4}, \eta_{4}\right\rangle \text {. }
$$

Now, according to 3.3.3 and 3.3.5 of [3], there is an element $\xi \in\left\langle 2, \eta_{4}, \eta_{4}\right\rangle$ such that $\xi \in\{p\}$, also $v \theta_{4} \in\{p\}$. Obviously $\xi-v \theta_{4}$ has $d$-invariant zero and filtration $\geqslant 6$ in $\pi_{33}$. From $E_{\infty}$ of the Adams spectral sequence [13], taking account of the corrections 3.3.6 and 3.3.7 of [3], $\xi-v \theta_{4} \in \eta \pi_{32}$. Now, since $v \theta_{3}=\eta \theta_{3}=0$, it follows that $\theta_{3} \xi=0$, and so $\theta_{3}\left\langle 2, \eta_{4}, \eta_{4}\right\rangle$ contains zero; since $\theta_{3} \eta_{4}$ has filtration $\geqslant 4$ in $\pi_{30}$ it must be zero so there is no indeterminacy, $\theta_{3}\left\langle 2, \eta_{4}, \eta_{4}\right\rangle=0$, and $\eta \eta_{4} \theta_{4}=0$.

LEMMA 4.2.

$$
\eta \beta=\kappa \eta_{5}
$$

Proof. By Lemma 3.2, $\eta \kappa \pi_{30}=0$, as $\pi_{30}$ is generated by $\theta_{4}$. Therefore

$$
\eta \beta=\eta\left\langle 2, \theta_{4}, \kappa\right\rangle=\left\langle\eta, 2, \theta_{4}\right\rangle \kappa=\eta_{5} \kappa+\eta \kappa \pi_{30}=\eta_{5} \kappa .
$$


LEMMA 4.3. There is an element $\zeta \in\left\{d_{1}\right\} \subseteq \pi_{32}$ such that $\eta \zeta=0$ and $\theta_{3} \zeta=\eta^{2} \gamma$.

Proof. There are relations in $\operatorname{Ext}_{A}(\mathbb{Z} / 2, \mathbb{Z} / 2)[\mathbf{2 0}]$,

$$
h_{1} g_{2}=h_{3} e_{1}, \quad h_{1} e_{1}=h_{3} d_{1}, \quad h_{2}^{2} n=h_{1} t
$$

Next from [13] and [6, Theorem 4.1] we see that $h_{1} e_{1}$ and $d_{1}$ are infinite cycles, but $\delta_{3} e_{1}=h_{1} t=h_{2}^{2} n$.

It is straightforward to check that there is an element $\zeta \in\left\{d_{1}\right\}$ such that $\eta \zeta=0$, see [13, p. 354] taking account of 3.3.4, 3.3.6 and 3.3.7 of [3]. We shall prove that $\theta_{3} \zeta$ and $\eta^{2} \gamma$ are equal by showing that they both lie in the bracket $\langle\sigma, v, v \xi\rangle \eta$, where $\xi$ is any element of $\{n\}$, and that this bracket consists of a single element.

From the above Ext relations we see that $\sigma \zeta \in\left\{h_{1} e_{1}\right\}$. The differential $\delta_{3} e_{1}=h_{2}^{2} n$ shows that if $\xi \in\{n\}$ then $v^{2} \xi=0$. Now Moss's theorem and a simple check on indeterminacy show that

$$
\langle v, v \xi, \eta\rangle \subseteq\left\{h_{1} e_{1}\right\}
$$

Now, as in $[3$, p. 316], pick $\tau$ in the set $\langle\eta, v, \kappa \bar{\kappa}\rangle \cap\{u\}$, then

$$
2 \tau \in 2\langle\eta, v, \kappa \bar{\kappa}\rangle=\langle 2, \eta, v\rangle \kappa \bar{\kappa}=0
$$

It now follows that the indeterminacy in the choice of $\tau$ is the element of order 2 in the image of $J$ and, for all choices of $\tau$, the $e$-invariant of $\eta \tau$ is zero. From [3, p. 316] we know that $\eta \tau$ and $2 \bar{\kappa}^{2}$ both lie in $\left\{h_{1} u\right\}$. It is clear that $2 \bar{\kappa}^{2}$ has $e$-invariant zero, and so we conclude that $\eta \tau=2 \bar{\kappa}^{2}$.

The indeterminacy of the set $\left\{h_{1} e_{1}\right\}$ is generated by $\bar{\sigma} \bar{\kappa} \in\left\{c_{1} g\right\}, \tau \in\{u\}$ and $\rho_{39} \in\left\{P^{4} h_{3}\right\}$ where $\rho_{39}$ is a generator of the image of $J$. Therefore there are integers $x, y, z$ such that

$$
\sigma \zeta+x \bar{\sigma} \bar{\kappa}+y \tau+z \rho_{39} \in\langle\nu, v \xi, \eta\rangle
$$

The $e$-invariant of $\sigma \bar{\kappa}$ is zero so, since $\pi_{27}$ is equal to the image of $J[3$, p. 355], this shows that $\sigma \bar{\kappa}=0$. Next we show that $\sigma \tau=0$; suppose not, then for filtration reasons $\sigma \tau \in\{g j\}$, and so $\eta \sigma \tau \neq 0$, see Theorem 3.1(iii). But we have shown that $\eta \tau=2 \bar{\kappa}^{2}, \sigma \bar{\kappa}=0$ and so $\eta \sigma \tau=0$. Finally a check on filtration shows that $\sigma \rho_{39}$ is zero and therefore

$$
\sigma^{2} \zeta \in \sigma\langle v, v \xi, \eta\rangle=\langle\sigma, v, v \xi\rangle \eta
$$

Now apply Moss's theorem and a simple check on indeterminacy to see that $\langle\sigma, v, v \xi\rangle \subseteq\left\{h_{3} e_{1}\right\}$. However $h_{1} g_{2}=h_{3} e_{1}$ and so there are integers $p$ and $q$ such that

$$
\begin{gathered}
\eta \gamma+4 p \alpha+q \delta \in\langle\sigma, v, v \xi\rangle, \quad \eta^{2} \gamma+q \eta \delta \in\langle\sigma, v, v \xi\rangle \eta, \\
\eta^{3} \gamma+q \eta^{2} \delta \in\langle\sigma, v, v \xi\rangle \eta^{2}
\end{gathered}
$$

Now $\eta^{3}=4 v, 4 \gamma=\theta_{3} \theta_{4}[3,3.5 .2]$ and so $\eta^{3} \gamma$ is zero. We have just proved that $\langle\sigma, v, v \xi\rangle \eta$ contains a multiple of $\sigma^{2}$ and therefore $\langle\sigma, v, v \xi\rangle \eta^{2}$ contains zero (since $\eta \sigma^{2}=0$, see $\left[22\right.$, p. 190]); in the proof of Lemma 3.5 we checked that $\eta^{2} \sigma \pi_{38}=0$ so 
this bracket has indeterminacy zero. Therefore $q \eta^{2} \delta=0$ and since $\eta^{2} \delta \neq 0$ by Theorem 3.1 we conclude that $q$ is even, $q \eta \delta=0$ and $\eta^{2} \gamma \in\langle\sigma, v, v \xi\rangle \eta$.

We have shown that $\eta^{2} \gamma$ and $\sigma^{2} \zeta$ are both elements of $\langle\sigma, v, v \xi\rangle \eta$ and we must now show that the indeterminacy of this bracket is zero. The only point which is non-trivial is the proof that $\eta \sigma \pi_{38}=0$. However it is easy to check that if $\eta \sigma \pi_{38} \neq 0$ then the only non-trivial element of $\eta \sigma \pi_{38}$ is $\delta$. But, as we have noted, $\eta^{2} \sigma \pi_{38}=0$ whereas $\eta \delta \neq 0$. Therefore $\langle\sigma, v, v \xi\rangle \eta$ contains a single element and $\eta^{2} \gamma=\sigma^{2} \zeta$ as required.

We now put Lemmas 4.1, 4.2 and 4.3 together to complete the proof of Lemma 2.3. By Moss's theorem, the differentials $\partial_{2} h_{4}=h_{0} h_{3}^{2}, \partial_{2} h_{5}=h_{0} h_{4}^{2}$ show that in filtration 3 of the Adams spectral sequence, $\left\langle\theta_{3}, 2, \theta_{4}\right\rangle$ is represented by $h_{4}^{3}+h_{3}^{2} h_{5}=0$. Therefore, using a simple check on indeterminacy, any element of $\left\langle\theta_{3}, 2, \theta_{4}\right\rangle$ has filtration $\geqslant 4$ and so this set is contained in the subgroup of $\pi_{45}$ generated by $2 \alpha, \beta, \eta \gamma, \delta$. Thus, by Lemma $4.1(\mathrm{i}), \eta_{4} \theta_{4}$ is in the subgroup of $\pi_{46}$ generated by $\eta \beta, \eta^{2} \gamma, \eta \delta$.

We checked, in the proof of Lemma 4.3, that $\eta^{3} \gamma=0$. We now verify that $\eta^{2} \beta=0$. By definition, $\beta \in\left\langle\kappa, \theta_{4}, 2\right\rangle$ so that

$$
\begin{aligned}
& \eta^{2} \beta \in \eta^{2}\left\langle\kappa, \theta_{4}, 2\right\rangle \\
& =\left\langle\eta^{2}, \kappa, \theta_{4}\right\rangle 2 \quad \text { since } \eta^{2} \kappa=0 \\
& =2\left\langle\eta^{2}, \kappa, \theta_{4}\right\rangle=\left\langle 2, \eta^{2}, \kappa\right\rangle \theta_{4} \subseteq \pi_{17} \theta_{4} .
\end{aligned}
$$

By Moss's theorem, $\left\langle 2, \eta^{2}, \kappa\right\rangle \cap\left\{h_{0} e_{0}\right\} \neq \varnothing$ and there is a relation in $\operatorname{Ext}_{A}(\mathbb{Z} / 2, \mathbb{Z} / 2), \quad h_{2} d_{0}=h_{0} e_{0}$. Now $e$ - and $d$-invariant considerations show that $v \kappa \in\left\langle 2, \eta^{2}, \kappa\right\rangle$ and so $0 \in\left\langle 2, \eta^{2}, \kappa\right\rangle$ and therefore $\theta_{4}\left\langle 2, \eta^{2}, \kappa\right\rangle=0$.

Now we have established that $\eta_{4} \theta_{4}=x \eta^{2} \gamma+y \eta \beta+z \eta \delta, x, y, z \in \mathbb{Z} / 2$, and the facts that $\eta \eta_{4} \theta_{4}=0, \eta^{2} \delta \neq 0$ (Theorem 3.1(ii), (iv)) show that $z=0$. Therefore using Lemmas 4.2 and 4.3 , we take $\psi_{1}=x \zeta$ and $\psi_{2}=y \eta_{5}$ so that

$$
\eta_{4} \theta_{4}=\theta_{3} \psi_{1}+\kappa \psi_{2} \text {. }
$$

\section{APPENDIX}

The vanishing of certain Toda brackets

Let $P_{n+1}$ be the truncated real projective space $\mathbb{R} P^{\infty} / \mathbb{R} P^{n}$ and let $i: S^{n+1} \rightarrow P_{n+1}$ be the inclusion of the bottom cell.

TheOREM A.1. Suppose that $\alpha \in \pi_{n}^{s}, \beta \in \pi_{j}^{s}$ with $j<n$ and $\alpha \beta=0$. If

$$
\beta \in \operatorname{ker}\left\{i_{*}: \pi_{j+n+1}^{s} S^{n+1} \longrightarrow \pi_{j+n+1}^{s} P_{n+1}\right\},
$$

then $0 \in\langle\alpha, \beta, \alpha\rangle$. 
Lemma 1.2 is an immediate corollary of this theorem.

There are two approaches to such results, one we give here and the other we intend to discuss in a future paper on the quadratic construction. The two approaches give slightly different results; using the quadratic construction we need additional hypotheses on $\alpha$, for example, $n$ is even, but can dispense with the hypothesis that $j<n$. Incidentally the proof we give here shows that the condition $j<n$ may be replaced by a weaker but much more messy condition.

We begin work on the proof of Theorem A.1. Let $V_{n+1}$ be the Stiefel manifold $\mathrm{O} / \mathrm{O}(n+1)$, so there is a fibration

$$
S^{n+1} \longrightarrow V_{n+1} \longrightarrow V_{n+2} \text {. }
$$

Let $u: P_{n+1} \rightarrow V_{n+1}$ be the usual inclusion, then the hypothesis that $j<n$ implies that $\pi_{j+n+1}^{s} S^{n+1}=\pi_{j+n+1} S^{n+1}$ and also that

$$
u_{*}: \pi_{j+n+1} P_{n+1} \longrightarrow \pi_{j+n+1} V_{n+1}
$$

is an isomorphism. Therefore

$$
\beta \in \operatorname{ker}\left\{\pi_{j+n+1} S^{n+1} \longrightarrow \pi_{j+n+1} V_{n+1}\right\}
$$

and so, from the long exact sequence of the above fibration, there is an element $\phi \in \pi_{j+n+2} V_{n+2}$ such that $\partial \phi=\beta$. Write $\xi \in \pi_{j+n+2} \mathrm{BO}(n+2)$ for the image of $\phi$ under the map $V_{n+2} \rightarrow \mathrm{BO}(n+2)$. We identify $\xi$ with a stably trivial vector bundle over $S^{j+n+2}$ and write $E$ for the sphere bundle of this vector bundle. Then $E$ has cell structure

$$
\left(S^{n+1} \cup_{\beta} e^{j+n+2}\right) \cup e^{2 n+j+3} .
$$

Lemma A.2. The sphere bundle $E$ has the same stable homotopy type as

$$
\left(S^{n+1} \cup_{\beta} e^{j+n+2}\right) \vee S^{2 n+j+3} .
$$

Proof. Since $E$ is a stably trivial sphere bundle over a sphere it is homeomorphic to a stably parallelizable manifold and so the attaching map of the top cell is stably trivial.

Now let $B G$ be the classifying space for stable spherical fibrations. Then for any $\mathrm{CW}$ complex $A,[S A, B G]$ is in one-one correspondence with the set $\left\{A, S^{0}\right\}$ of stable homotopy classes of stable maps $A \rightarrow S^{0}$. So given $\alpha \in \pi_{n}^{s}$ such that $\alpha \beta=0$, there exists a stable map

$$
S^{n} \cup_{\beta} e^{n+j+1} \longrightarrow S^{0}
$$

extending $\alpha$. This now corresponds to a map $f_{X}: X \rightarrow B G$, where $X$ is the $j+n+2$ skeleton of $E$. Using Lemma A.2 and the fact that $B G$ is an infinite loop space this map $f_{X}$ extends to a map $f: E \rightarrow B G$; write $T(f)$ for the Thom spectrum determined by the corresponding spherical fibration over $E$, normalized so that the inclusion of a fibre determines an element in $\pi_{0}^{s} T(f)$. 


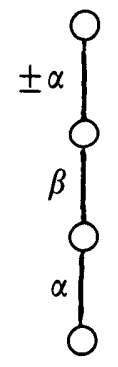

Fig. 11

Lemma A.3. The stable cell diagram of $T(f)$ is as shown in Figure 11.

Proof. The $n+j+2$ skeleton $X$ of $E$ is a suspension, and a standard result on Thom complexes over suspensions shows that $T\left(f_{X}\right)$ is the mapping cone of the stable map

$$
S^{n} \cup_{\beta} e^{n+j+1} \longrightarrow S^{0}
$$

corresponding to $f_{X}$. We conclude that the cell diagram for $T(f)$ is as shown in Figure 12 , for some $\gamma \in \pi_{n}^{s}$. The diagonal map $E \rightarrow E \times E$ induces a stable map

$$
\Delta: T(f) \longrightarrow E_{+} \wedge T(f)
$$

where $E_{+}$means $E$ with a disjoint base point adjoined. We can assume $n \geqslant 1$ and then write $\Phi: H^{t}(E ; \mathbb{Z}) \rightarrow H^{t}(T(f) ; \mathbb{Z})$ for the Thom isomorphism, so that the homomorphism $\Delta^{*}$, in integral cohomology, is given by the formula

$$
\Delta^{*}(x \otimes \Phi(y))=\Phi(x y), \quad x, y \in H^{*}(E ; \mathbb{Z})
$$

Now write $p: E_{+} \rightarrow S^{n+j+2}$ for a stable map of degree one in integral cohomology; such a map exists by Lemma A.2. This gives a stable map

$$
q=(p \wedge 1) \Delta: T(f) \longrightarrow S^{j+n+2} T(f),
$$

and this in turn gives a stable map

$$
r: T(f) / T(f)^{(n+1)} \longrightarrow S^{n+j+2} T(f)^{(n+1)}
$$

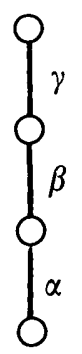

FIG. 12 
Here we write $Y^{(k)}$ for the $k$-skeleton of the $\mathrm{CW}$ spectrum $Y$. A cohomology calculation shows that $r$ is an equivalence, but

$$
\begin{gathered}
T(f) / T(f)^{(n+1)}=S^{j+n+2} \cup_{y} e^{2 n+j+3} \\
S^{n+j+2} T(f)^{(n+1)}=S^{j+n+2} \cup_{\alpha} e^{2 n+j+3}
\end{gathered}
$$

and so $\alpha= \pm \gamma$.

This proves Lemma A.3; Theorem A.1 is an easy deduction.

\section{References}

1. J. F. ADAMS, 'On the non-existence of elements of Hopf invariant one', Ann. of Math., 72 (1960), 20-104.

2. J. F. ADAMS, 'On the groups $J(X)$-IV', Topology, 5 (1966), 21-71.

3. M. G. Barratt, M. E. Mahowald and M. C. TANGora, 'Some differentials in the Adams spectral sequence-II', Topology, 9 (1970), 309-316.

4. M. G. Barratt, J. D. S. Jones and M. E. Mahowald, 'The Kervaire invariant problem', Proceedings of the Northwestern homotopy theory conference, Contemporary Mathematics 19 (American Mathematical Society, Providence, 1983), pp. 9-23.

5. M. G. BarRatT, J. D. S. Jones and M. E. MahOWALD, 'The Kervaire invariant and the Hopf invariant', preprint, Warwick University, 1982.

6. R. BRUNER, 'A new differential in the Adams spectral sequence', preprint, Wayne State University, 1983.

7. D. M. Davis and M. E. Mahowald, ' $v_{1}$ and $v_{2}$ periodicity in stable homotopy theory', Amer. J. Math., 103 (1981), 615-659.

8. J. D. S. JONES, 'The Kervaire invariant of extended power manifolds', Topology, 17 (1978), 249-266.

9. M. E. MAHOWALD, 'On the order of the image of $J$ ', Topology, 6 (1967), 371-378.

10. M. E. MAHOWALD, 'The metastable homotopy of $S^{n}$, Memoir 72 (American Mathematical Society, Providence, 1967).

11. M. E. MAHOWALD, 'Some remarks on the Kervaire invariant problem from a homotopy point of view', Proceedings of Symposia in Pure Mathematics 22 (American Mathematical Society, Providence, 1971).

12. M. E. MahowaLd, 'A new infinite family in ${ }_{2} \pi_{*}^{s}$ ', Topology, 16 (1977), 249-256.

13. M. E. Mahowald and M. C. TANGora, 'Some differentials in the Adams spectral sequence', Topology, 6 (1967), 349-369.

14. M. E. MAhOWALD and M. C. TANGora, 'An infinite subalgebra of $\operatorname{Ext}_{A}\left(\mathbb{Z}_{2}, \mathbb{Z}_{2}\right)$ ', Trans. Amer. Math. Soc., 132 (1968), 263-274.

15. R. J. Milgram, 'Symmetries and operations in homotopy theory', Proceedings of Symposia in Pure Mathematics 22 (American Mathematical Society, Providence, 1971), pp. 203-210.

16. R. J. Milgram, 'Group representations and the Adams spectral sequence', Pacific J. Math., 41 (1972), $157-182$.

17. R. E. Mosher and M. C. TANGora, Cohomology operations and applications in homotopy theory (Harper and Row, New York, 1968).

18. R. M. F. Moss, 'Secondary compositions and the Adams spectral sequence', Math. Z., 115 (1970), 283-310.

19. F. P. Peterson and N. Stein, 'Secondary cohomology operations: two formulas', Amer. J. Math., 81 (1959), 281-305.

20. M. C. TANGORA, 'On the cohomology of the Steenrod algebra', Math. Z., 116 (1970), 18-64.

21. M. C. TANGora, 'Some extension questions in the Adams spectral sequence', Proceedings of the Advanced Study Institute on algebraic topology, August 1970, Vol. III (Aarhuus University, Aarhuus, 1970).

22. H. TODA, Composition methods in homotopy groups of spheres (University Press, Princeton, 1962).

\author{
(Barratt and Mahowald) \\ Mathematics Department, \\ Northwestern University, \\ Evanston, \\ Illinois 60201, \\ U.S.A.
}

\author{
(Jones) \\ Mathematics Institute, \\ University of :Warwick, \\ Coventry CV4 7AL.
}

